\title{
The impact of body composition parameters on ipilimumab toxicity in metastatic melanoma
}

\author{
L. Daly ${ }^{1}$, A. O’Reilly ${ }^{2}$, P. Donnelan, S. Cushen ${ }^{1}$, D. Woodlock ${ }^{2}$, D.G. Power ${ }^{3}$ and A.M. Ryan ${ }^{1}$ \\ ${ }^{1}$ School of Food and Nutritional Sciences, University College Cork, Ireland, ${ }^{2}$ Department of Medical Oncology, \\ University Hospital Galway, Ireland and ${ }^{3}$ Department of Medical Oncology Mercy \& Cork University Hospitals, \\ Cork, Ireland
}

Body composition has emerged as an important predictor of chemotherapeutic drug efficacy and toxicity ${ }^{(1,2,3)}$. Ipilimumab (Ipi) is a monoclonal antibody that inhibits cyototoxic T lymphocyte antigen-4 (CTLA-4), an inhibitory receptor expressed on T lymphocytes. This inhibiton allows $\mathrm{T}$ cell immune activation against melanoma antigen and has been shown to improve overall survival in patients with advanced melanoma. Immune-related toxicity can result as a consequence of Ipi mode of action. There are no validated predictive biomarkers of Ipi efficacy or toxicity.

The aim of this study was to evaluate if body composition, specifically sarcopenia, as assessed by computed tomography (CT) can predict toxicity to Ipi in metastatic melanoma. Patients with metastatic melanoma, treated with Ipi at two university teaching hospitals between 2009-2015 were included. Skeletal muscle cross-sectional area at L3 was measured using by CT using OsiriX ${ }^{\circledR}$ software (Pixmeo, Geneva, Switzerland). Sarcopenia was defined using sex specific published cut-offs ${ }^{(4)}$. Toxicity was recorded using the Common Terminology Criteria for Adverse Events (CTCAE) v4.0.

Sixty-six patients were included in this study, 38 were male $(58 \%)$. In total $68 \%(n=45)$ were overweight or obese by WHO standards $\left(\right.$ BMI $\left.>25 \mathrm{~kg} / \mathrm{m}^{2}\right)$. Twenty four $(36.4 \%)$ were sarcopenic at baseline, and $17 \%(\mathrm{n}=11)$ had sarcopenic obesity. Overall $71 \%$ completed all 4 cycles of treatment and $15.2 \%$ experienced early cessation of treatment as a result of significant toxicity. In total, $57.6 \%$ experienced an immune-related adverse event, the most common being dermatologic toxicity (rash and pruritus) (35\%), followed by gastrointestinal (GI) toxicities (31.8\%, including diarrhoea and colitis). Overall $70 \%$ of patients experienced Grade (gr) $1-2$ toxicities while $40 \cdot 9 \%$ (27) experienced gr 3-4 toxicities.

We observed no significant difference in the prevalence of GI, dermatologic, or endocrine immune-related events between sarcopenic and non-sarcopenic patients across all 4 cycles of the drug [(20.8 \% vs $38 \cdot 1 \%(p=0 \cdot 241), 33 \cdot 3 \%$ vs $8 \cdot 1 \%(p=0 \cdot 904), 8 \cdot 3 \%$ vs $11.9 \%(\mathrm{p}=1.00)$ respectively]. We noted that patients with a BMI $>30 \mathrm{~kg} / \mathrm{m}^{2}[16(24.6 \%)]$, had a significantly higher incidence of GI toxicities $(81.2 \%$ vs $38.8 \%, p=0.008)$, including diarrhoea $(62.5 \%$ vs $20.4 \%, p=0.004$, gr $3-4$ diarrhoea $12.5 \%$ vs $0 \%$, $\mathrm{p}=0.001)$, nausea $(56.2 \%$ vs $12.2 \%, \mathrm{p}=0.001)$ and colitis $(68.6 \%$ vs $42.9 \%, \mathrm{p}=0.01)$

Sarcopenic patients were identified as being more susceptible to early treatment cessation; only $58 \%$ of sarcopenic patients received the full 4 cycles of treatment compared with $83 \%$ of non-sarcopenic patients $(p=0.09)$. Overall $33 \%$ of sarcopenic patients received $<2$ cycles of treatment compared with $11.9 \%$ of non sarcopenic patients $(p=0.053)$

We found no significant difference in treatment toxicity between sarcopenic and non-sarcopenic patients, and this may partially be explained by the pharmacokinetics of ipilimumab, as it is not protein-bound drug. Previous studies, which have demonstrated an increase in toxicity in sarcopenic patients have been carried out in protein bound drugs e.g. Docetaxel ${ }^{(1)}$, Sorafenib ${ }^{(2)}$, Epirubicin $^{(3)}$. We conclude that Ipilimumab toxicity is not increased in patients with sarcopenia, however sarcopenic patients tend to spend less time on treatment. This study may have implications for Ipi outcomes and further data will be presented.

1. Cushen S, Power DG, Teo My et al. (2014) Am J Clin Onco [Epub ahead of print

2. Prado CM, Lima I, Baracos V et al. (2010) Cancer Chemother Pharmacol 67, 93-101

3. Mir O, Coriat R, Blanchet B et al. (2012) Plos Biol 5, 37563

4. Mourtzakis M, Prado C.M, Lieffer JR et al. (2008) Appl Physiol, Nutr Metab 33, 997-1006 